\title{
Flexible heat shields deployed by centrifugal force
}

DOI:

10.1016/j.actaastro.2018.06.021

\section{Document Version}

Accepted author manuscript

Link to publication record in Manchester Research Explorer

\section{Citation for published version (APA):}

Wu, R., Roberts, P., Soutis, C., \& Diver, C. (2018). Flexible heat shields deployed by centrifugal force. Acta Astronautica, 152, 78-87. https://doi.org/10.1016/j.actaastro.2018.06.021

\section{Published in:}

Acta Astronautica

\section{Citing this paper}

Please note that where the full-text provided on Manchester Research Explorer is the Author Accepted Manuscript or Proof version this may differ from the final Published version. If citing, it is advised that you check and use the publisher's definitive version.

\section{General rights}

Copyright and moral rights for the publications made accessible in the Research Explorer are retained by the authors and/or other copyright owners and it is a condition of accessing publications that users recognise and abide by the legal requirements associated with these rights.

\section{Takedown policy}

If you believe that this document breaches copyright please refer to the University of Manchester's Takedown Procedures [http://man.ac.uk/04Y6Bo] or contact uml.scholarlycommunications@manchester.ac.uk providing relevant details, so we can investigate your claim.

\section{OPEN ACCESS}




\title{
Flexible Heat Shields Deployed by Centrifugal Force
}

\author{
Rui Wu ${ }^{\mathrm{a}, *}$, Peter C.E. Roberts ${ }^{\mathrm{a}}$, Constantinos Soutis ${ }^{\mathrm{b}}$, Carl Diver ${ }^{\mathrm{a}}$ \\ ${ }^{a}$ School of Mechanical, Aerospace and Civil Engineering, UK \\ ${ }^{b}$ The University of Manchester Aerospace Research Institute, UK
}

\begin{abstract}
Atmospheric entry aerodynamic decelerators which also provide thermal protection do not scale well for smaller payloads (e.g. CubeSat) or where the planets atmosphere is significantly less dense than the Earth's (e.g. Mars entry). Both cases require heat shields larger than can be accommodated either within the launch vehicle fairing, or within acceptable payload volumes, so deployable shields are required. Unlike previous designs proposed to fulfil this requirement like inflatable structures or deployable solid mechanisms, the presented research addresses this by utilising inertial force, or specifically, centrifugal force generated from autorotation to deploy and stiffen a flexible heat shield. Structural dynamic analyses including the trajectory simulation on a CubeSat sized system has shown that the autorotation and deployment form a closed loop which reliably leads to an equilibrium of deployment, while the heat shield is near fully deployed at altitudes higher than $30 \mathrm{~km}$ with tolerable spin rate $(<6 \mathrm{rps})$ and oscillation. Thermal analysis suggests that a front surface temperature reduction of $100 \mathrm{~K}$ is achievable on a CubeSat sized vehicle as unlike inflatable structures, no thermal insulation is needed around the flexible material. This design concept can realise a lightweight, compact and concise entry system.
\end{abstract}

Keywords:

heat shield, entry vehicle, deployable aerodynamic decelerator, centrifugal deployment, origami, aeroelastic oscillation, thermal protection, morphing

\section{Nomenclature}

$a \quad=\quad$ ratio of the first stage diameter in the second stage generatrix length

$a_{d e c}=$ deceleration $\left(\mathrm{m} / \mathrm{s}^{2}\right)$

$C_{D} \quad=$ drag coefficient

$d \quad=$ heat shield thickness $(\mathrm{m})$

e $\quad=$ surface emissivity

$f_{\text {struc. }}=$ structural natural frequency $(\mathrm{Hz})$

$f_{\text {pitch }}=$ aerodynamic pitching frequency $(\mathrm{Hz})$

$f_{\omega} \quad=$ spin rate of autorotation in $\mathrm{Hz}(\mathrm{Hz})$

$I_{\text {deploy }}=$ shell's angular inertia associated with its deploying motion $\left(\mathrm{kg} \cdot \mathrm{m}^{2}\right)$

\footnotetext{
* Corresponding author

Email address: rui.wu@manchester.ac.uk (Rui Wu)
} 


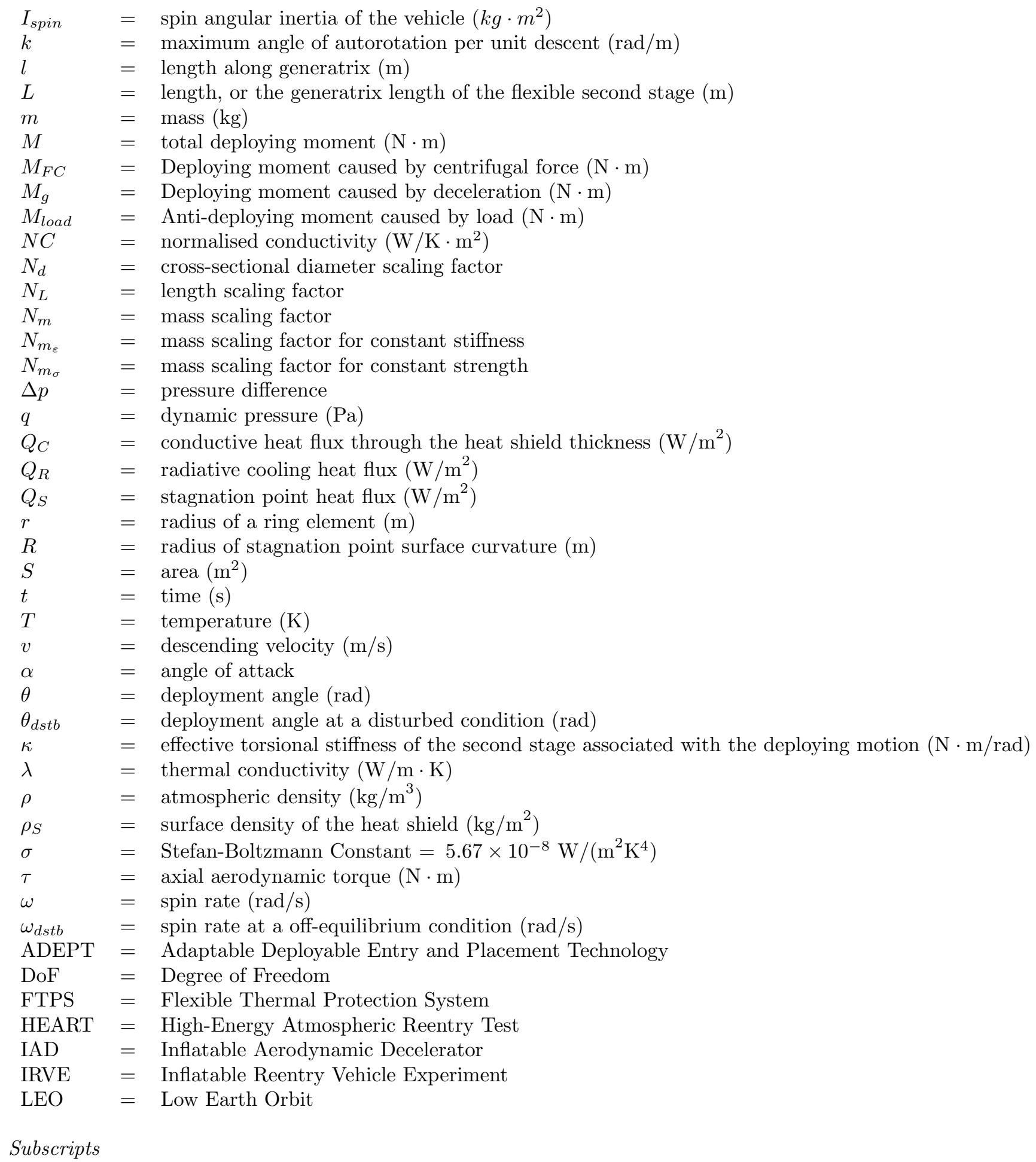


front $=$ front surface

back $=$ back surface

\section{Introduction}

Atmospheric entry reduces the kinetic and potential energy of a spacecraft, allowing soft landing on a planetary surface. However, the entry process depends on the atmospheric properties, and the entry system design has to be adapted for various planetary environments as well as payloads. Current Mars entry systems usually consist of a rigid blunt aeroshell that decelerates the payload from hypersonic to supersonic speed, and a parachute that deploys at supersonic velocities and lower altitudes. However, Mars entry requires a low ballistic ratio, $\mathrm{m} / S C_{D}$, to effectively slow down the vehicle through the thin Martian atmosphere, meaning the size of rigid aeroshells is approaching the fairing capacity of existing launching vehicles and future missions with heavier robotic systems or manned vehicles are not achievable using the current rigid aeroshell technology. The quest for a new system that could enable heavier payloads to be landed on Mars calls for a deployable aeroshell that can be stowed compactly in a launch vehicle while creating large drag area when deployed [1, 2, 3, 4, 5. Meanwhile, a deployable aeroshell could also address the demand for a CubeSat-sized recoverable platform for in-orbit scientific experiments since a low ballistic ratio is favourable for thermal control. This is challenging due to the high surface curvature and surface area to volume ratio of small vehicles. Furthermore, the low ballistic ratio could waive the necessity for a parachute due to the low terminal velocity. This also enables a single decelerator system that is inherently safe since it burns up in the atmosphere in case of deployment failure. A few efforts have been reported in this area 6 , $7,8,8$.

To realise a deployable aeroshell, various designs were investigated during the 1960s and the 1970s. Although very little research has been reported from the mid 1970s to the mid 2000s, the recent demand for more advanced missions outside the existing systems' performance envelope has promoted renewed interest. The Inflatable Aerodynamic Decelerator (IAD) is the most studied concept, which has seen various adaptations in recent years [9, 10, 11, 12, 13, 14, such as the Inflatable Reentry Vehicle Experiment-4 (IRVE-4) vehicle shown in Fig. 1a 13. An IAD forms a blunt shape using flexible pressure chambers inflated by either internal or external gas sources. It utilises the superior tensile strength and stiffness of the pressure chambers made from a flexible fabric, as well as the compressive properties of gas, which is the ultimate lightweight and flexible material. In addition to IAD, there have been a few published concepts on conventional umbrellalike mechanisms consisting of rigid structural elements connected by revolving or sliding joints 15, 16, 17, such as the Adaptable Deployable Entry and Placement Technology (ADEPT) concept shown in Fig. 1b 18. Such a structure could allow shape morphing during the entry, and therefore, enable trajectory control [6, 19.

On the other hand, while both the mechanically deployable structures and the inflatable structures are deployed and stabilised by elastic forces from solid or gaseous materials, a structure can also be deployed and achieve stiffness using inertia forces. For example, the increased bending stiffness of a spinning helicopter rotor is a well-studied phenomenon of centrifugal stiffening [20]. Meanwhile, studies have also shown that centrifugal force can be utilised to deploy large flexible space structures. Although the lack in internal and external damping is a challenge for flexible structures in an orbital environment, the Anamya-2 experiment has successfully demonstrated the centrifugal deployment of a 20-metre wide space mirror [21, 22, 23]. The application of inertia forces 


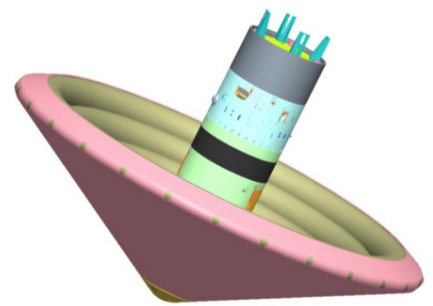

(a)

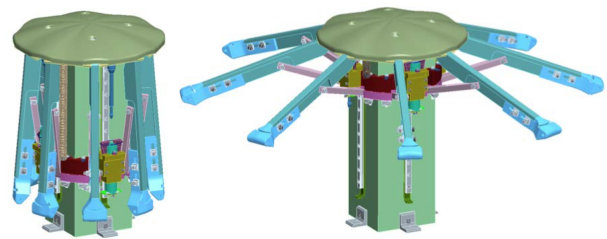

(b)

Figure 1: Two types of deployable entry system concepts, (a) the IRVE-4 vehicle with an IAD [13, (b) the ADEPT concept with a mechanically deployable heat shield [18.

could open up new types of entry systems that may potentially benefit from the advantages of centrifugal deployment, including being lightweight, compact and needing low power for deployment [24, 25].

The presented study shows that it is feasible to use centrifugal force to deploy and stabilise an aerodynamic decelerator by utilising a flexible morphing heat shield that passively regulates its deployment and centrifugal force. Specifically, $\$ 2$ provides a very basic comparison between the mass of elastically and centrifugally supported beams, $\$ 3$ discusses the design concept in detail and proposes a baseline CubeSat sized vehicle. Structural dynamic analyses using analytical and numerical methods, as well as a trajectory simulation on the baseline vehicle are included in \$4. The thermal analyses in $\$ 5$ shows that the proposed concept also has advantages in terms of thermal control.

\section{Structural Advantages of Centrifugal Deployment and Stiffening}

Structural mass is a major concern for space systems. Here a simplified comparison is made between two types of structures deployed by elastic and centrifugal forces respectively according to the behaviour of a basic structural element. For an elastically deployed structure, consider a cantilever beam under a transverse aerodynamic load with a unit load density (thus a constant dynamic pressure), as shown in Fig. 2a Consider the nature of aerodynamic load, the overall load on the beam can be assumed to be $\propto N_{L}^{2}$, where $N_{L}$ is the scaling factor of the beam's length. The scaling factor of a parameter means the ratio between the values of the parameter before and after scaling. Assuming constant material modulus, when scaling the size of the beam up, a constant deflected shape, thus a constant stiffness can be achieved by keeping a constant aeroelastic-bending parameter according to the linear beam theory: $N_{d}^{4} / N_{L}^{4}=1$ (according to the aeroelastic bending similitude parameter from [26]), where $N_{d}$ is the scaling factor of the beam's cross sectional diameter. Similarly, keeping the maximum normal stress constant requires $N_{L}^{3} N_{d} / N_{d}^{4}=1$ (according to Eq. 5-13 from [27). Therefore, the mass scaling factor $N_{m} \propto N_{d}^{2} N_{L}$ for achieving constant stiffness $\left(N_{m_{\varepsilon}}\right)$ and strength $\left(N_{m_{\sigma}}\right)$ become:

$$
N_{m_{\varepsilon}} \propto N_{d}^{2} N_{L} \propto N_{L}^{3}, \quad N_{m_{\sigma}} \propto N_{d}^{2} N_{L} \propto N_{L}^{3}
$$

Meanwhile, instead of a cantilever beam, the most efficient way for a rod to carry load by elasticity is to align the load along the rod axis such as in a truss structure, as shown in Fig. 2b, so 
the material is evenly stressed. To analyse a rod under such a condition, the previous model is used with the distributed load changed to a force along the rod with its magnitude $\propto N_{L}{ }^{2}$. Ignoring the effect of buckling for a compression scenario, the similitude of stiffness and strength can be achieved if the strain/stress in the rod remains constant during scaling, which requires: $N_{L}{ }^{2} / N_{d}^{2}=1$. Then the mass scaling factors become $N_{m_{\varepsilon}}=N_{m_{\sigma}} \propto N_{d}{ }^{3}$.

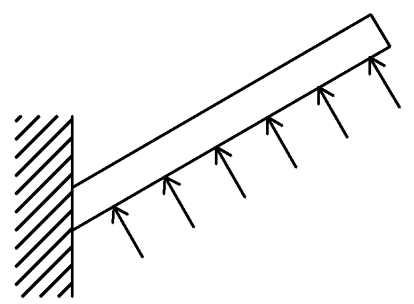

(a)

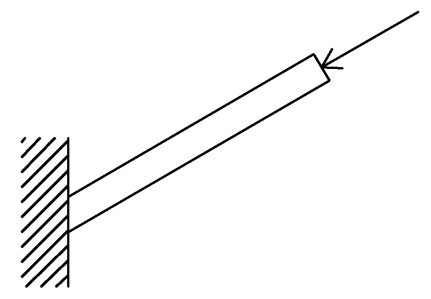

(b)

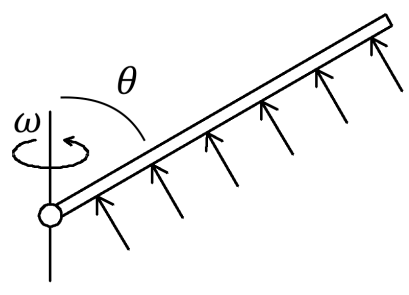

(c)

Figure 2: Schematic view of three types of basic structural elements, (a) cantilever beam supported by elastic force, (b) axially loaded rod, (c) spinning rod supported by centrifugal force.

For a structure deployed by centrifugal force, consider a rod with a mass of $m$ and a length of $L$ hinged on one end and spins around an axis that passes through the hinged point at a spin rate of $\omega$, as shown in Fig. 2c. Now apply a uniformly distributed lateral aerodynamic load with a unit magnitude, and ignore its elastic deformation which has minor effect on the deployment. Statically the equilibrium can be reached when the deploying moment from centrifugal force, $M_{F C}$, equals the anti-deploying moment from the load, $M_{\text {load }}$, under a certain deployment angle of $\theta$, which is the angle between the rod and the spin axis. Since the total aerodynamic load is proportional to $L^{2}$ as mentioned above, the moment from the aerodynamic load becomes $M_{\text {load }} \propto L^{3}$. Meanwhile, the centrifugal force can be expressed as:

$$
m \omega^{2} \sin (\theta) L
$$

Where the term $\sin (\theta) L$ is proportional to the radius of the circular motion. Then from equilibrium, we have:

$$
\frac{M_{\text {load }}}{M_{F C}}=1 \propto \frac{L^{3}}{\left(m \omega^{2} \sin (\theta) L \cdot \cos (\theta) L\right)}
$$

Where the term $\cos (\theta) L$ is proportional to the moment arm of centrifugal force. Then the mass scaling factor for stiffness (or in other words, the condition to achieve a constant deflection $\theta$ ) can be calculated by solving Eq. 3 for $m$ while assuming constant $\omega$ and $\theta$. The result gives $m \propto L$. The scaling factor for strength can be determined based on the tensile stress in the rod induced by centrifugal force. According to Eq. 2, this requires:

$$
N_{m_{\sigma}} \omega^{2} \sin (\theta) N_{L} / N_{d}^{2} \propto 1
$$

Assuming a uniform scaling with $N_{L} / N_{d}=1$, we have $N_{m_{\sigma}} \propto N_{L}$. The results are summarised in Table 1

From Table 1 it can be seen that in order to carry a load, the mass of a cantilever beam or an axially loaded bar has to increase more rapidly with size than a centrifugally deployed rod. 
Table 1: Comparison between a cantilever beam, an axially loaded rod and centrifugally deployed rod.

\begin{tabular}{|c|c|c|c|}
\hline Condition & $\begin{array}{l}\text { Stabilisation } \\
\text { force }\end{array}$ & $\begin{array}{l}\text { Mass scaling factor for } \\
\text { constant stiffness }\end{array}$ & $\begin{array}{l}\text { Mass scaling factor for } \\
\text { constant strength }\end{array}$ \\
\hline $\begin{array}{l}\text { Cantilever beam } \\
\text { Axially loaded rod }\end{array}$ & Elastic & $\begin{array}{l}N_{m_{\varepsilon}} \propto N_{L}^{3} \\
N_{m_{\varepsilon}} \propto N_{L}^{3}\end{array}$ & $\begin{array}{l}N_{m_{\sigma}} \propto N_{L}{ }^{3} \\
N_{m_{\sigma}} \propto N_{L}{ }^{3}\end{array}$ \\
\hline Centrifugally deployed rod & Centrifugal & $N_{m_{\varepsilon}} \propto N_{L}$ & $N_{m_{\sigma}} \propto N_{L}$ \\
\hline
\end{tabular}

Therefore, to deploy a large heat shield, centrifugal deployment could be advantageous to elastic deployment in terms of structural mass. Meanwhile, Table 1 only provides the dependence of mass on linear dimension, while the effects of other parameters on a centrifugally deployed rod are provided by Eq. 3 . For instance, a higher attainable spin rate $\omega$ or a lower required deployment angle $\theta$ leads to lower mass.

Besides the mass saving potential for a large vehicle, centrifugal deployment could also benefit a small vehicle, such as a CubeSat-sized re-entry vehicle, since it eliminates the actuators or pressure vessels in inflatable or mechanically deployed heat shields and allows a concise system that is selfregulated (as further discussed in \$3).

In addition, since a centrifugally deployed structure could minimise the dependence on elastic support and be designed to carry solely tension, it can be constructed from materials with low flexural rigidity. Therefore, it provides more freedom for packaging and is likely to achieve higher compactness than a solid deployable mechanism.

\section{Design Concept}

The core of the design concept for the entry heat shield is a flexible thin shell with a spiral characteristic and a uniform surface density (Fig. 3a). The shape generates an axial aerodynamic roll-torque that causes autorotation during descent, thus the spiral characteristic of the shape determines the spin rate at certain descent velocity. Meanwhile, the autorotation produces a centrifugal force that stiffens the shell but also flattens it (Fig. 3b), thus the spin rate determines the shell's deployment under certain external conditions and hence the roll-torque that is produced. Therefore, the deployment, the characteristic of the spiral shape and the spin rate form a closed loop that regulate each other. Since the deployment of the shell can flatten the spiral shape and reduce the induced roll-torque, the roll-torque is self regulating, leading to an equilibrium spin rate.

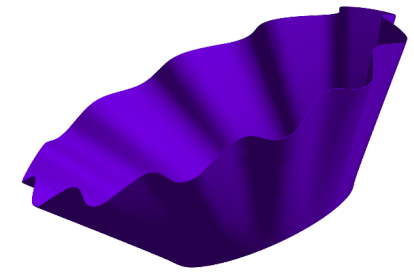

(a)

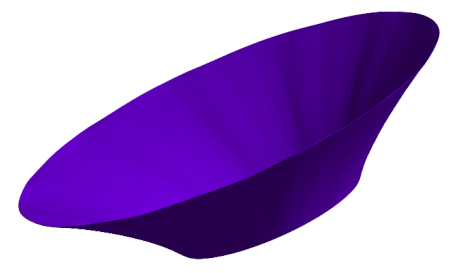

(b)

Figure 3: Shapes of the shell at different deployed states according to FE (Finite Element) simulations, (a) a partially deployed state that tends to produce high roll-torques, (b) a near fully deployed state that leads to low roll-torques and stabilises spin rate. 
The spiral shape shown in Fig. 3 is based on an origami pattern, which allows the heat shield to be packed into a cylindrical shell with a diameter close to the rigid nose cone of the payload vessel, as shown in Fig. 4. The packing method is similar to "Wrap-Rib" described in [28]. The ridges and valleys of the spiral shape are aligned along the folding lines, and the pattern could be tailored to adapt to different spiral shapes. The spiral characteristics formed by those ridges and valleys leads to an aerodynamic roll-torque as discussed in Fig. 3. Since the origami guarantees that no folding lines pass across the ridges and valleys of the shell during folding, the creep of material (when the shell is packed) will not significantly change the spiral characteristics of the shape. This could enable longer mission duration and allow packaging prior to the spacecraft assembly.

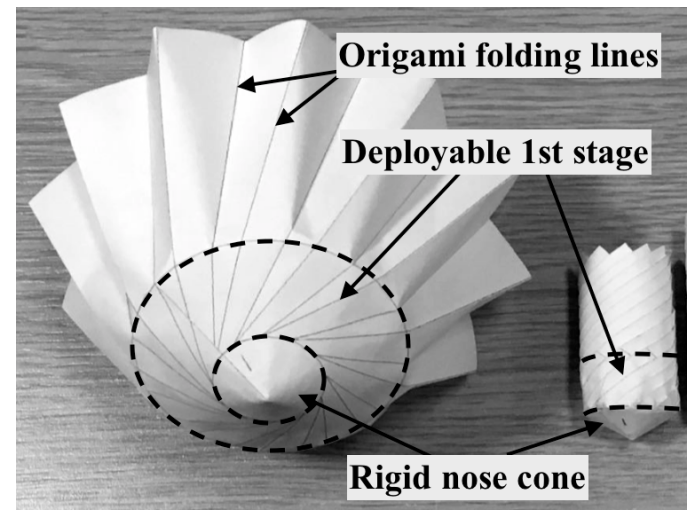

Figure 4: Deployed (left) and packed (right) configurations of paper prototypes, showing that the origami pattern upon which the spiral shape is based allows the heat shield to be packed into a cylindrical shell. The first stage that can rigidify after deployment, as well as the rigid nose cone of the payload vessel are labelled.

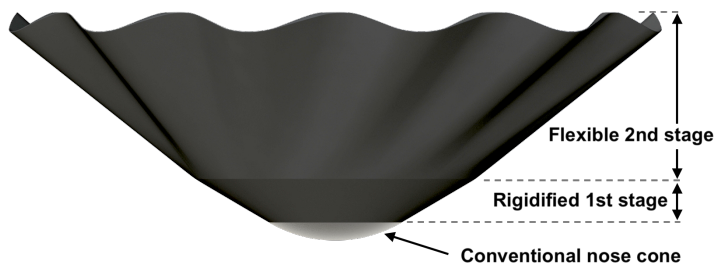

(a)

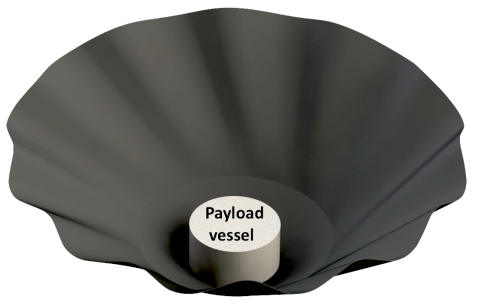

(b)

Figure 5: Conceptual design of a vehicle with centrifugally deployed heat shield, (a) front view showing the basic components, (b) tilted view showing the payload vessel behind the nose cone.

The heat shield is divided into two stages, as shown in Fig. 4. The whole shell can be unpacked when the first stage deploys, which may be achieved by an inflatable or a mechanically deployable structure. The first stage becomes rigid after deployment, then the flexible second stage can deploy by centrifugal force as discussed above and increase the drag area significantly. The first stage not only enables the initial unpacking, but also assists the second stage deployment since it increases the diameter and therefore, the centrifugal force on the second stage. In the absence of such a first 
stage, the flexible second stage could be entangled near the spin axis where the centrifugal force is low as shown later in the discussion associated with Fig. 77. The payload vessel is located behind the rigid nose cone, which can be a conventional thermal protection system (Fig. 5). Although the concept contains an elastically deployed first stage, the design could still benefit from the advantages of centrifugal deployment of the second stage as discussed in $\$ 2$ and potentially achieve higher packing ratio and lower mass.

In terms of thermal control, such a heat shield requires a FTPS (Flexible Thermal Protection System) to protect the structure from aerothermodynamic heating while allowing packing and shape morphing. The development of IADs has raised similar demand, and various material systems including ceramic and organic fibres as well as flexible insulating materials have been tested in lab environment and in flight tests [29, 30. Meanwhile, unlike the airtight pressure chambers of IADs, the proposed heat shield is a single wall structure that potentially allows high surface temperatures, which not only eliminates the requirement for heat insulation, but also allows effective radiative cooling from the aft surface, which is analysed in $\S 5$.

For instance, the heat shield can be constructed using stitched woven ceramic fabrics where the spiral shape is formed using the stitched seams or local reinforcements along the origami pattern, or thermally protected elastomers for lower energy missions (e.g. suborbital), where the spiral shape is formed by material elasticity. Meanwhile, the edge (shoulder) of the flexible shell can be rounded by a fold hem created by folding and stitching similar to the ADEPT concept to prevent excessive local heating [31].

\section{Structural Analysis}

Operation of the heat shield begins after the initial deployment, which rigidises the first stage and unpacks the second stage. Therefore, the structural analyses presented here are focused on the behaviour of the flexible second stage during the ballistic entry. To assess the static and dynamic structural response associated with the deploying-folding motion of the flexible stage, analytical analysis based on a simplified model as well as FE simulations with higher fidelity were conducted. A trajectory simulator was also developed, which accounts for the simplified structural dynamic model and the 3 DoF (Degree of Freedom) trajectory (assuming the shield points stably into the flow at zero angle of attack).

A sample re-entry mission from Low Earth Orbit (LEO) is used to characterise the trajectory as well as structural dynamic behaviours of a CubeSat-sized baseline vehicle. The baseline vehicle has a total mass of $1.3 \mathrm{~kg}$, a cone semi-vertex angle of $60^{\circ}$, a base diameter of $0.7 \mathrm{~m}$ when fully deployed and $0.125 \mathrm{~m}$ when fully packed.

\subsection{Deployment}

In this section, analytical equations are derived to predict the spin rate and deployment angle at certain static conditions, which show satisfactory agreement with FE simulations. The equations also demonstrate the ability of the shell to recover after an instantaneous disturbance, and the effects of different design parameters are illustrated.

To conduct analytical analysis, a simplified geometrical model similar to the origami shown in Fig. 4 is made, which consists of panels that are hinged together along the origami's folding lines, and allows an axisymmetrical deploying-folding motion with one degree of freedom. Since this motion is unique, the shapes and orientations of the panels can be determined by geometry using 
solely deployment angle $\theta$, then the aerodynamic pressures on the panels can be calculated using the Newtonian method.

Similar to Eq. 3, deployment requires:

$$
M=M_{F_{C}}-M_{\text {load }}+M_{g} \geq 0
$$

$M_{F_{C}}$ can be evaluated by dividing the shell into ring elements with the mass of $d m$ (Fig. 6), then integrating the moment generated by the centrifugal force from each ring element. Each ring element is assumed to be circular and is perpendicular to the vehicle axis with a certain radius $r=a L+l \cdot \sin (\theta)$. The term $\cos (\theta) \cdot l$ in the following equation is the moment arm of the centrifugal force.

$$
M_{F_{C}}=\int_{0}^{L} d m \cdot \omega^{2} r \cos (\theta) l
$$

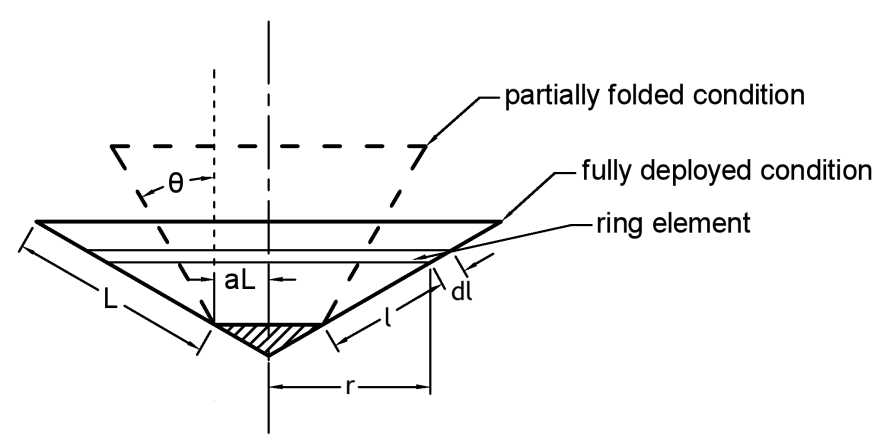

Figure 6: Vehicle schematic view, showing the major dimensions of the rigid first stage and the flexible second stage, as well as the ring element used in calculation.

Note that the heat shield with a certain spiral shape behaves like an unloaded wind turbine in autorotation, which has a maximum spin rate proportional to descending velocity:

$$
\omega=k \cdot v
$$

Where $k$ is determined by the characteristics of the spiral shape and depends on $L, a$, and $\theta$ (those variables are illustrated in the vehicle schematic view shown in Fig. 6). It is numerically evaluated using the simplified geometrical model and Newtonian aerodynamics.

Meanwhile, the mass of the ring element, $d m$ can be expressed as below, where the term $2 \pi$. $\left[\sin \left(60^{\circ}\right) \cdot l+a L\right]$ is the length of the heat shield material around the ring element:

$$
d m=d S \cdot \rho_{S}=2 \pi\left[\sin \left(60^{\circ}\right) l+a L\right] \rho_{S} \cdot d l
$$

According to Newtonian Aerodynamics, the aerodynamic pressure normal to a surface is [32]:

$$
\Delta p=\rho v^{2} \sin ^{2}(\alpha)
$$

Then similar to Eq. 6, $M_{\text {load }}$ can be evaluated by integrating the anti-deploying moment caused by the aerodynamic load, which has a moment arm of $l$, while assuming the second stage is a conical surface with a semi-vertex angle of $\theta$. 


$$
M_{\text {load }}=\int \rho v^{2} \sin ^{2}(\theta) l \cdot d S
$$

Where $d S=2 \pi r \cdot d l$ is the surface area of the ring element, and $\alpha$ (in Eq. 9 is approximated by $\theta$ for simplicity. Higher fidelity analysis has also been carried out to evaluate Eq. 10 using $\alpha$ determined from the geometrical model, which yields similar results for $\theta$ within $\pi / 6 \sim \pi / 3$ $\left(30^{\circ} \sim 60^{\circ}\right)$. Similarly, $M_{g}$ can be evaluated by integrating the deploying moment from the inertial force induced by deceleration, which has a moment $\operatorname{arm}$ of $\sin (\theta) \cdot l$ :

$$
M_{g}=\int_{0}^{L} d m \cdot a_{\operatorname{dec}} \sin (\theta) l
$$

The deceleration $a_{d e c}$ can be expressed as below, where $C_{D}$ can be evaluated using Eq. 9 as a function of $\theta$.

$$
a_{\text {dec }}=\frac{q S C_{D}}{m}
$$

By substituting Eqs. 6] 12 into Eq. 5, the deployment criteria can be derived. In order to save computational resource, the expression is simplified by setting parameters based on a baseline vehicle design: $a=0.577$, and the mass scaling law $m \propto L^{3}$, then some near-linear terms containing $\theta$ are linearised. The result is shown as Eq. 13 According to the discussions associated with Table 2 , the linear regression has no significant effect on calculation results, but can considerably reduce the computation time.

$$
M \approx v^{2}\left[k^{2} \rho_{S} L(0.573 \theta+0.788) \cos (\theta)-\rho(1.01 \theta-0.431) \sin (\theta)\right] \geq 0
$$

From Eq. 13 , it can be seen that a thinner atmosphere, a higher heat shield surface density, a larger heat shield size, and a higher $k$ are beneficial to the deployment, as all leads to a higher value of $M$.

Meanwhile, $\omega$ can be determined by substituting Eq. 7into Eq. 13. The result is shown below as Eq. 14. For a certain vehicle design, it can be used to calculate the required spin rate $\omega$ to achieve a deployment angle $\theta$ under a certain condition of $q$ by evaluating the term on the right hand side of the equation. However, this equation does not provide information on whether the overall equilibrium is achieved, since the overall equilibrium also depends on the roll-torque generated at such a spin rate, or in other words, the dependence of $k$ on other factors, which is numerically analysed in 4.2 .

$$
\omega \approx \sqrt{\frac{\tan (\theta) q(2.01 \theta-0.861)}{\rho_{S} L(0.573 \theta+0.788)}}
$$

To validate Eqs. 13 and 14 , a series of $\mathrm{FE}$ simulations have been conducted to determine whether the predicted deployment angles can be achieved under certain conditions. A flexible second stage is modelled as shown in Fig. 3 which is based on the baseline design of a CubeSat sized vehicle that has a base diameter of $0.7 \mathrm{~m}$, a cone semi-vertex angle of $60^{\circ}$ when fully deployed, a total mass of $1.3 \mathrm{~kg}$, an $a$ value of 0.577 , and a surface density $\rho_{S}$ of $1.1 \mathrm{~kg} / \mathrm{m}^{2}$. The effect of rotation, deceleration and Newtonian aerodynamic load are taken into account, and static nonlinear simulations are carried out. The results are shown in Table 2 , which also corresponds the 
Table 2: Comparison between the deployment angles predicted by the analytical equations before and after linear regression as well as FE simulations.

\begin{tabular}{llllll}
\hline $\begin{array}{l}\text { Dynamic } \\
\text { pressure }\end{array}$ & $\begin{array}{l}\text { Spin } \\
\text { rate }\end{array}$ & $\begin{array}{l}\text { Deploy. angle } \\
\text { (analytical) }\end{array}$ & $\begin{array}{l}\text { Deploy. angle } \\
\text { (regression) }\end{array}$ & $\begin{array}{l}\text { Deploy. angle } \\
\text { (FE) }\end{array}$ & Condition \\
\hline $180 \mathrm{~Pa}$ & $5.2 \mathrm{rps}$ & $58^{\circ}$ & $60^{\circ}$ (full deploy) & $56^{\circ}$ & Peak dynamic pressure \\
$26 \mathrm{~Pa}$ & $1.7 \mathrm{rps}$ & $55^{\circ}$ & $55^{\circ}$ & $52^{\circ}$ & $35 \mathrm{~km}$ altitude \\
$31 \mathrm{~Pa}$ & $1.6 \mathrm{rps}$ & $50^{\circ}$ & $49^{\circ}$ & $46^{\circ}$ & $24 \mathrm{~km}$ altitude \\
$43 \mathrm{~Pa}$ & $1.4 \mathrm{rps}$ & $44^{\circ}$ & $42^{\circ}$ & $38^{\circ}$ & $12 \mathrm{~km}$ altitude \\
$60 \mathrm{~Pa}$ & $1.2 \mathrm{rps}$ & $36^{\circ}$ & $35^{\circ}$ & $30^{\circ}$ & Sea level \\
\hline
\end{tabular}

simulated conditions to the flight regimes predicted by the trajectory simulation discussed in 4.2 The results from the equations with linear regressions are also reported.

It can be seen that the predictions from analytical equations are not significantly changed by linear regression. Meanwhile, in comparison with FE simulations, the equations over-predict the deployment angles by no more than $6^{\circ}$ throughout descent, which is acceptable for providing a preliminary understanding about the flight performance.

Equations 13 and 14 assume a static condition where the total deploying moment on the shell is zero. However, disturbances during the flight such as abrupt change in the atmospheric density or structural oscillation could rapidly change the deployment angle and lead to off-equilibrium conditions. The stability of deployment, or in other words, the ability of the shell to recover from disturbance and regain equilibrium is critical to the survivability of the vehicle. For example, instability could occur after an abrupt disturbance in case more than one deployment angle could satisfy the deployment criteria under the disturbed condition, e.g. under certain scenario the shell may rest near the spin axis where the centrifugal force is low. To analyse the possibility of instability, Eq. 13 can be rearranged to evaluate the deploying moment at a certain off-equilibrium condition:

$$
M \propto \omega_{d s t b}^{2} \rho_{S} L \cdot \cos (\theta)\left(0.573 \theta_{d s t b}+0.788\right)-q \cdot \sin \left(\theta_{d s t b}\right)\left(2.01 \theta_{d s t b}-0.861\right)
$$

Assuming the disturbance is instantaneous, $\omega_{d s t b}$ can be related to $\omega$, the static spin rate before the disturbance predicted by Eq. 14, according to the conservation of angular momentum: $\omega \cdot I_{\text {spin }}(\theta)=\omega_{d s t b} \cdot I_{\text {spin }}\left(\theta_{d s t b}\right)$, where $I_{\text {spin }}$ is a function of $\theta$ and can be determined based on the simplified geometrical model while ignoring the angular inertia of payload (considering the relatively low radius of the payload vessel).

Then the recovering effect at a disturbed condition can be characterised by the angular acceleration of the shell's deploying motion: $\ddot{\theta}=M / I_{\text {deploy }}$, where $I_{\text {deploy }}$ is evaluated according to the simplified geometrical model. Thus static stability requires $\ddot{\theta} /\left(\theta_{d s t b}-\theta\right)<0$, and a $\ddot{\theta}$ with higher absolute value means more rapid recovery. The dependences of $\ddot{\theta}$ on $a$ and $L$ are shown in Fig. 7. where the horizontal axis is the instantaneous deployment angle after the disturbance. Generally, the design shows that deployment is statically stable, though this does not guarantee dynamic stability (as discussed later in $\S 4.2$ and $\S 4.3$ ). It also worth noticing that the analysis only considers the rolling motion and deploying-folding motion, while pitch and yaw instabilities are not considered here.

The recovering effect of heat shields with different sizes and with two different initial equilibrium deployment angles are shown in Fig. $7 \mathrm{a}$ and $7 \mathrm{~b}$. It can be seen that $\ddot{\theta}$ reduces with increasing heat shield size as a result of increasing shell inertia. Analysis that is not presented here has also shown 
that the shell surface density has a similar impact. Meanwhile, a higher initial deployment angle before the disturbance leads to a higher recovering effect due to the higher loads. As shown by Fig. $7 \mathrm{c}$ the size of the first stage has a non-monotonic impact on $\ddot{\theta}$. An $a$ value of 0.577 , which is chosen for the baseline design, gives a near optimum overall recovering effect, while instability could occur when the shell is pushed to a fully folded state of $\theta=0^{\circ}$ if $a$ was zero.

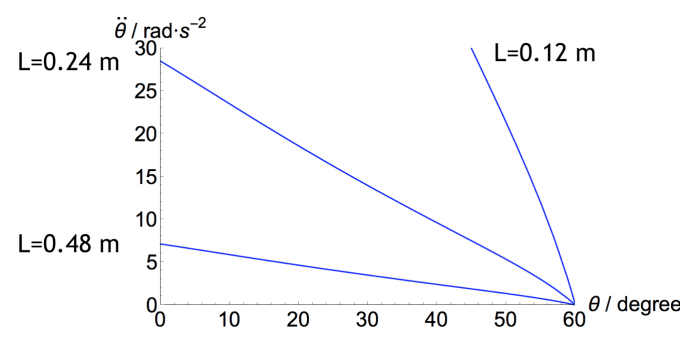

(a)

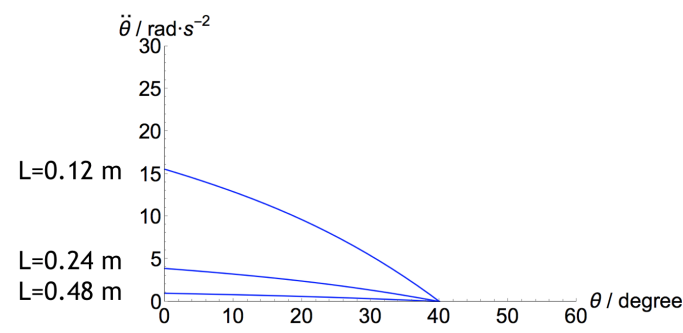

(b)

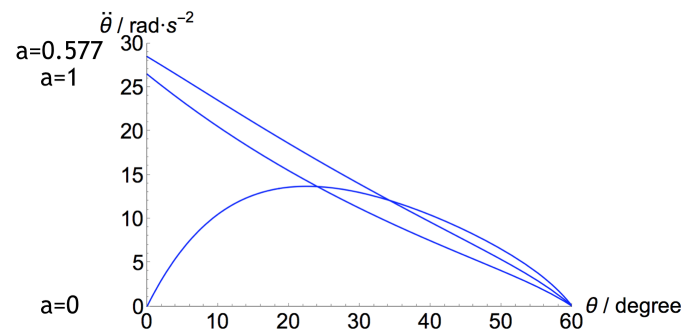

(c)

Figure 7: The effects of design parameters on the shell's ability to recover from instantaneous disturbance of deployment angle under a unit dynamic pressure, where a high (absolute value) deploying angular acceleration means rapid recovery, (a) the effect of size under a $\mathbf{6 0} 0^{\circ}$ initial deployment angle before the disturbance, (b) the effect of size under an initial deployment angle of $\mathbf{4 0 ^ { \circ }}$, (c) the effect of first stage size with a constant overall size.

\subsection{Trajectory Simulation}

The equations describing the axisymmetrical deploying-folding motion of the heat shield's flexible second stage are derived in $\$ 4.1$. In this section, those equations are used to simulate the dynamic structural response during a 3 DoF trajectory simulation.

The previous equations can be used to determine the deployment angle according to spin rate and other internal and external conditions, while how the deployment influences the spiral shape and thus the spin rate is still needed to simulate the closed loop of the heat shield. Therefore, the axial aerodynamic torque $\tau$ is derived as a function of $\theta, \omega, v$ and $q$ using the simplified geometrical model and Newtonian aerodynamics and assuming a zero angle of attack. The result generally shows that the heat shield is similar to an unloaded wind turbine: spin rate under a condition of $\tau=0$ is proportional to axial velocity. Meanwhile, the spin rate at $\tau=0$ drops linearly to zero as the deployment angle increases to a fully deployed condition. Such a relation can support a passive proportional control as described in $\$ 3$.

The deployment angle can be calculated by integrating the instantaneous acceleration of deploying motion with respect to time, as shown below. The first integral (deploying angular velocity) is 
set to zero every time when the maximum deployment angle is reached to represent the mechanical constraint on deployment.

$$
\theta=\iint \frac{M}{I_{\text {deploy }}} \cdot d t d t
$$

Similarly, the spin rate of autorotation can be determined:

$$
\omega=\frac{\int \tau \cdot d t}{I_{\text {spin }}}
$$

The structural model is then incorporated into a 3 DoF trajectory simulator based on Simulink. The simulator is developed using Newton's second law and describes the point mass dynamics of the vehicle in various reference systems. It assumes zero angle of attack and ignores the gyroscopic effects from spinning. Such simplification is made as the presented study is focused on the heat shield's deployment rather than the full flight dynamics of the vehicle.

The re-entry of a CubeSat sized vehicle with the baseline design from a $145 \mathrm{~km}$ orbit at zero flight path angle is simulated, and the results are shown in Fig. 8. It can be seen that the deployment angle is above $50^{\circ}$ through most of the descent until the heat shield reaches an altitude below 25 $\mathrm{km}$. This can be explained by Eq. 13, since the low atmospheric density at high altitude leads to easy deployment, while at low altitude the shell starts to fold as the spiral shape is unable to provide sufficient spin rate (insufficient value of $k$ ). The reason for the deployment angle to drop at higher altitude especially during the simulation time from $300 \mathrm{~s}$ to $900 \mathrm{~s}$ is that the aerodynamic torque at such high altitude is too low to accelerate autorotation. This phenomenon can be prevented by adding an initial spin before the re-entry.

Meanwhile, structural oscillation that causes rapid fluctuations in deployment angle can be seen in the figure. The oscillation's frequency varies within $0.1 \mathrm{~Hz}-10 \mathrm{~Hz}$ simultaneously with dynamic pressure, and is discussed in 4.3 . According to the simulation, the peak amplitude of the oscillation gradually changes during the descent and is always below $6^{\circ}$, and the oscillation leads to fluctuations of axial deceleration with a peak amplitude of below $0.05 \mathrm{~g}$ at high altitude and 0.3 $\mathrm{g}$ at the peak dynamic pressure. Figure 8 has also shown the fluctuations of spin rate, which is a result of the conservation of angular momentum when the heat shield flutters. The oscillation is a result of the system's coupled dynamic behaviours, and is not the focus of the presented study. Nevertheless, it worth noticing that no structural damping is modelled in the simulator, thus there is likely to be less oscillation in reality where damping exist.

As shown by Eq. 14, the equilibrium spin rate is proportional to the dynamic pressure at a certain deployment angle. In Fig. 8 it can be seen that the spin rate increases and then drops during the first $1800 \mathrm{~s}$ of flight, which is a result of changing dynamic pressure. From $1800 \mathrm{~s}$, the change of spin rate is insignificant due to the generally constant dynamic pressure.

It should be noted that the simulated vehicle enters subsonic flight at $\sim 1720 \mathrm{~s}$, which is when the spin rate is dropped to $\sim 2 \mathrm{rps}$, and deployment angle starts to decrease from $60^{\circ}$ (Fig. 8). Since the simulator is developed using Newtonian aerodynamics, which is not suitable for subsonic speed, the simulator only provides a rough estimation of the flight after $1720 \mathrm{~s}$.

It also worth noticing that the simulation model does not account for the deploying/antideploying effect from the heat shield material's elasticity, which is expected to be minor as the objective of this design is to eliminate the reliance on elasticity and take advantage of centrifugal deployment. The most eminent effect may occur when the heat shield is near fully deployed (at higher altitude): since flattening a pre-folded shield made of thick fabric may require a relatively 


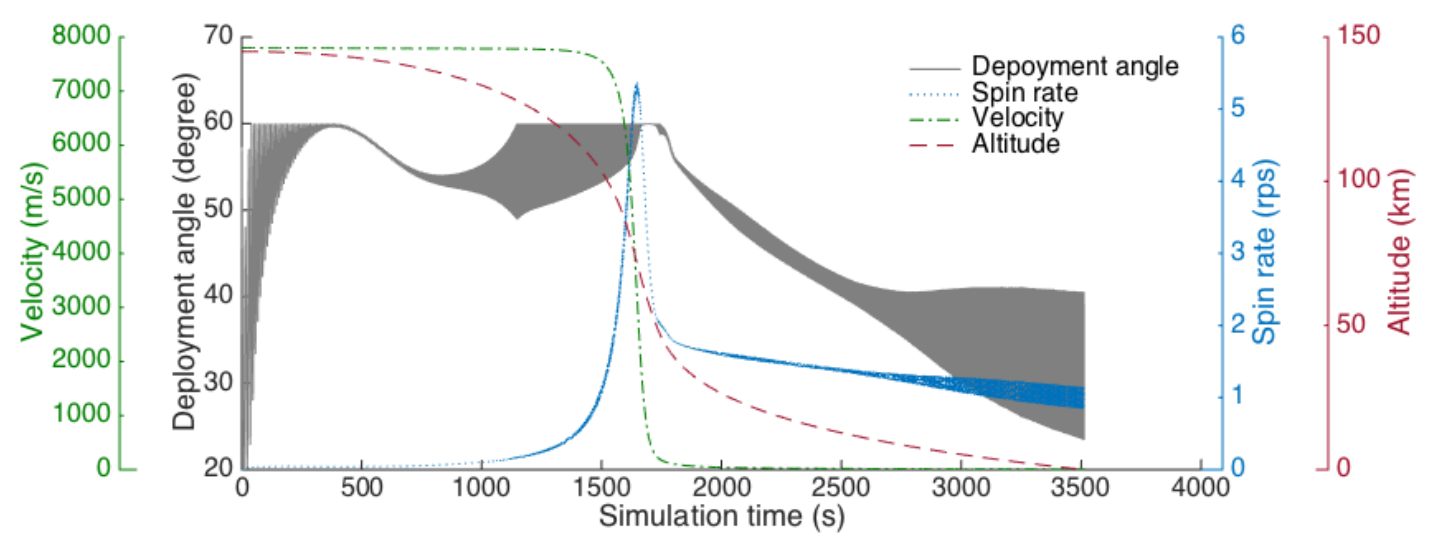

(a)

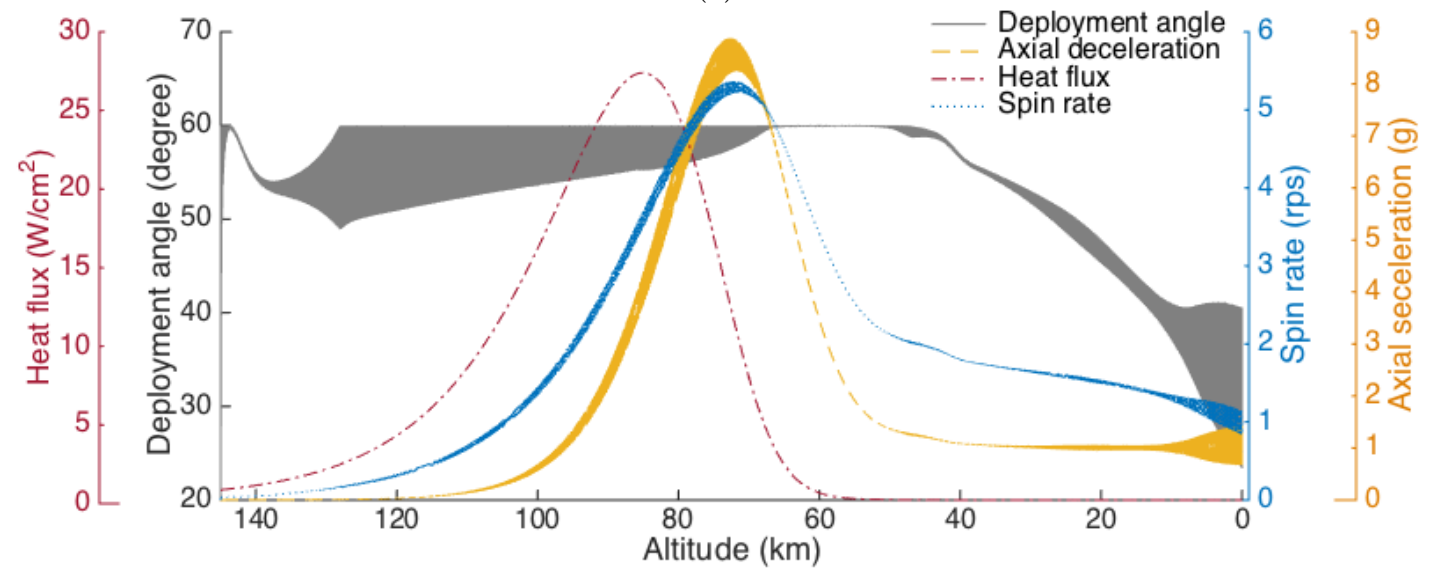

(b)

Figure 8: Trajectory simulation results on a CubeSat sized vehicle with baseline design plotted against (a) simulation time, (b) altitude, showing $>\mathbf{5 0}^{\circ}$ deployment angle before reaching $25 \mathrm{~km}$ altitude accompanied by structural oscillation.

high fabric tension, the shield will tend to have a lower deployment angle. However, a less-deployed shape leads to an increased spin rate due to the aerodynamic roll-torque, which will eventually deploy the shield to a higher extent.

\subsection{Structural Oscillation}

To avoid resonance, the structural natural frequency of the flexible second stage must not overlap with the spin rate and the aerodynamic pitching frequency. In this section, those three frequencies are analytically assessed, and the former two are also analysed by FE simulations.

The structural natural frequency associated with the shell's deploying-folding motion can be calculated using Eq. 18 below, where $\kappa$, the torsional stiffness of the shell associated with the oscillation, can be evaluated by differentiating the total deploying moment $M$ with respect to 
deployment angle $\theta: \kappa=\partial M / \partial \theta$. This equation has close agreement with the oscillation frequency observed in the trajectory simulation. The full equation is not shown here due to its complexity.

$$
f_{\text {struc. }}=\frac{1}{2 \pi} \sqrt{\frac{\kappa}{I_{\text {deploy }}}}=F\left(\theta, q, \omega, \rho_{S}, L, m, a\right)
$$

To validate Eq. 18 , the calculations are compared to the oscillating frequencies observed in the FE transient structural analyses under various conditions as predicted by the trajectory simulator, as shown in Table 3. The conditions are: high altitude at $1350 \mathrm{~s}$ (No. 1); peak heating at 1580 s (No. 2); peak dynamic pressure at $1620 \mathrm{~s}$ (No. 3); near fully deployed at low altitude at 1800 $\mathrm{s}$ (No. 4); partially deployed at sea level (No. 5). The shell is assumed to be made of a flexible material with a thickness of $1 \mathrm{~mm}$, a modulus of $5 \mathrm{MPa}$, and a density of $1.1 \mathrm{~g} / \mathrm{cm}^{3}$ according to the baseline design. The nonlinear FE transient structural analyses are carried out in ANSYS with the inertia force (centrifugal and deceleration) and aerodynamic force included, and the shell is divided into $>10,000$ shell element. The simulation provides a stabilised oscillation of $\sim 3 \mathrm{~s}$, and a variable time-step solver is used with the maximum time-step set to $1 / 150 \mathrm{~s}$. Running cases with varied shell material modulus also show that the effect of elasticity is neglectable, proving that the oscillation frequency is determined by dynamics.

Although the FE simulation uses a flexible model with a much higher degrees of freedom, the oscillations are always based on the deformation associated with deploying-folding motion. Therefore, as shown by Table 3, Eq. 18 has satisfactory agreement with the FE simulations except for conditions No. 1 and No. 5. The reason for these under-predictions is that the analytical model ignores the effect of elasticity, while as No. 1 and No. 5 are lightly loaded conditions (indicated by the low spin rates and deployment angles), the structural behaviours are dominated by the elasticity rather than the external/centrifugal load.

Table 3: Structural natural frequency from analytical analyses and FE simulations.

\begin{tabular}{llllllll}
\hline No. & $\begin{array}{l}\text { Dynamic } \\
\text { Pressure }\end{array}$ & $\begin{array}{l}\text { Spin } \\
\text { rate }\end{array}$ & $\begin{array}{l}\text { Decele- } \\
\text { ration }\end{array}$ & $\begin{array}{l}\text { Deploy. } \\
\text { angle }\end{array}$ & Eq. 18 & $\begin{array}{l}\text { FE simu- } \\
\text { lation }\end{array}$ & Differ. \\
\hline 1 & $1.5 \mathrm{~Pa}$ & $0.43 \mathrm{rps}$ & $0.0 \mathrm{~g}$ & $57^{\circ}$ & $0.77 \mathrm{~Hz}$ & $1.6 \mathrm{~Hz}$ & $-52 \%$ \\
2 & $100 \mathrm{~Pa}$ & $3.6 \mathrm{rps}$ & $4.1 \mathrm{~g}$ & $58^{\circ}$ & $6.4 \mathrm{~Hz}$ & $5.7 \mathrm{~Hz}$ & $12 \%$ \\
3 & $180 \mathrm{~Pa}$ & $5.2 \mathrm{rps}$ & $8.0 \mathrm{~g}$ & $60^{\circ}$ & $9.0 \mathrm{~Hz}$ & $9.5 \mathrm{~Hz}$ & $5 \%$ \\
4 & $26 \mathrm{~Pa}$ & $1.8 \mathrm{rps}$ & $1.0 \mathrm{~g}$ & $56^{\circ}$ & $3.2 \mathrm{~Hz}$ & $3.2 \mathrm{~Hz}$ & $0 \%$ \\
5 & $60 \mathrm{~Pa}$ & $1.2 \mathrm{rps}$ & $1.0 \mathrm{~g}$ & $35^{\circ}$ & $3.2 \mathrm{~Hz}$ & $4.2 \mathrm{~Hz}$ & $-24 \%$ \\
\hline
\end{tabular}

Equation 18 can be simplified to a function of $q$ and $L$, by assuming $a=0.577$, a fully deployed condition with $\theta=60^{\circ}$ (which is close to the shell's condition throughout high-speed regime), the mass scaling law based on the baseline design $m=94 L^{3}$, and the shell thickness (thus, surface density) proportional to vehicle size $\rho_{S}=4.58 \mathrm{~L}$. The result is below:

$$
f_{\text {struc. }}=0.160 \sqrt{\frac{q}{L^{2}}}
$$

Similarly, the spin rate can be simplified to:

$$
f_{\omega}=0.0928 \sqrt{\frac{q}{L^{2}}}
$$


The aerodynamic pitching frequency can be solved using Newtonian Aerodynamics according to literature 33. Consider a fully deployed heat shield, which is the case during most of the descent, and ignore the angular inertia of payload, which is minor in comparison with the deployed heat shield, the pitching frequency can be expressed as below with minor approximations:

$$
f_{\text {pitch }}=0.574 \sqrt{\frac{q}{L^{2}}}
$$

Combining Eqs. 19, 20 and 21, the ratios between the three frequencies for a vehicle with $a=0.577$ at a fully deployed equilibrium condition:

$$
f_{\omega}: f_{\text {struc. }}: f_{\text {pitch }}=0.58: 1: 3.6
$$

It worth noticing that Eq. 22 is valid regardless of vehicle size and dynamic pressure. Meanwhile, the frequency ratios can be tuned by adjusting the value of $a$. Therefore, the preliminary analyses presented here shows that the vehicle has designable and scalable dynamic behaviour.

\section{Thermal Analysis}

To provide a reference for the vehicle's thermal condition, the stagnation point heat flux is evaluated using the Sutton and Graves Equation shown below that accounts for convective heat transfer in non-reacting laminar flow 33, 34. The radiative heating is neglected due to the low entry velocity from LEO as well as the small vehicle size [32.

$$
Q_{S}=1.83 \times 10^{-4} v^{3} \sqrt{\frac{\rho}{R}} \quad\left(\mathrm{~W} / \mathrm{m}^{2}\right)
$$

Where atmospheric density $\rho$ has unit $\mathrm{kg} / \mathrm{m}^{3}$, stagnation point radius of curvature $R$ is in $\mathrm{m}$, velocity $v$ is in $\mathrm{m} / \mathrm{s}$, and the constant $1.83 \times 10^{-4}$ has dimension of $\mathrm{kg}^{0.5} \mathrm{~m}^{-1}$. Equation 23 is incorporated into the trajectory simulator, which predicts that, as shown in Fig. 8b, at the simulation time of $1540 \mathrm{~s}$ and an altitude of $87.5 \mathrm{~km}$, the heat shield experiences a peak heat flux of $27 \mathrm{~W} / \mathrm{cm}^{2}$, which is similar to the peak heat flux on NASA's High-Energy Atmospheric Reentry Test (HEART) vehicle, which utilises an IAD 35. Since the pressure chamber material of IAD requires a maximum operating temperature of $250^{\circ} \mathrm{C}$ to maintain air tightness [36, the FTPS in the HEART vehicle design contains insulating layers and hot gas barriers with a total surface density of $3-4 \mathrm{~kg} / \mathrm{m}^{2}$, while the surface density of the temperature resisting front layers is approximately 1 $\mathrm{kg} / \mathrm{m}^{2}$ (estimated based on the properties of $3 \mathrm{M}^{\mathrm{TM}}{ }^{\mathrm{N}}$ extel ${ }^{\mathrm{TM}} 440 \mathrm{BF} 20$ fabric) [35. This is close to $1.1 \mathrm{~kg} / \mathrm{m}^{2}$, the heat shield surface density of the presented CubeSat sized baseline design.

In comparison with IAD or conventional rigid heat shields, the centrifugally deployed heat shield has advantage in terms of thermal control. First of all, the requirement on thermal and hot gas insulation can be eliminated since the heat shield is a single wall structure with no payload or temperature-sensitive structures attached onto the aft surface, which leads to a lightweight and compact system. Meanwhile, the aft surface may effectively participate in the radiative cooling due to its high temperature, which helps reduce the overall temperature. A simple analysis is conducted to assess this effect.

Consider a high temperature non-ablating wall under thermal equilibrium, the front surface aerodynamic heating $Q_{S}$, the front surface radiative cooling $Q_{R_{\text {front }}}$ and the heat conducting through the heat shield thickness $Q_{C}$ should satisfy the relation: $Q_{S}=Q_{R_{\text {front }}}+Q_{C}$, while the backside 
radiative cooling $Q_{R_{b a c k}}$ is in equilibrium with $Q_{C}$, thus $Q_{C}=Q_{R_{b a c k}}$. Therefore, from the thermal transmission equations, we have:

$$
\begin{gathered}
Q_{S}=\sigma e_{\text {front }} T_{\text {front }}{ }^{4}+\frac{\lambda\left(T_{\text {front }}-T_{\text {back }}\right)}{d} \\
\frac{\lambda\left(T_{\text {front }}-T_{\text {back }}\right)}{d}=\sigma e_{\text {back }} T_{\text {back }}{ }^{4}
\end{gathered}
$$

Note that $\lambda$ and $d$ have similar effect on the equations, and can be substituted by a normalised conductivity: $N C=\lambda / d$. Then the front surface temperature $T_{\text {front }}$ can be resolved, which is a complicated function of $Q_{S}, e_{\text {front }}, e_{\text {back }}$, and $N C$. Assume $Q_{S}=27 \mathrm{~W} / \mathrm{cm}^{2}$ according to the trajectory simulation, $e_{\text {front }}=1$ as the worst case scenario for backside radiative cooling, then $T_{\text {front }}$ can be plotted against $e_{\text {back }}$ and $N C$, as shown in Fig. 9.

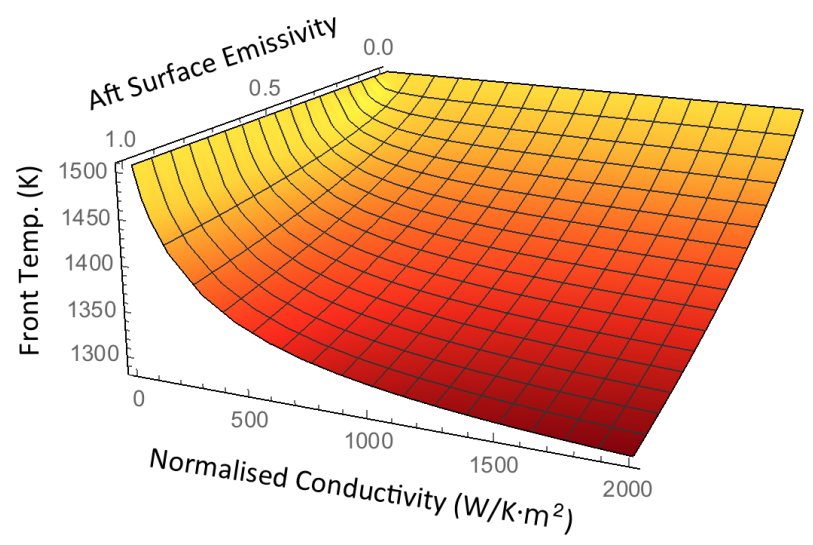

Figure 9: Prediction of the front surface temperature of an equilibrium non-ablating wall under $27 \mathbf{W} / \mathbf{c m}^{2}$ heating against aft surface emissivity and the wall's normalised conductivity, showing that temperature reduction can be achieved by aft surface radiative cooling.

Figure 9 shows that considerable reductions of front surface temperature can be achieved if the heat could be effectively conducted through the shell and radiated from the aft surface. For instance, the front surface temperature of an adiabatic wall with only front surface radiative cooling is $1506 \mathrm{~K}$, which can be reduced to $1397 \mathrm{~K}$ when $e_{\text {back }}=0.5, N C=500 \mathrm{~W} / \mathrm{K} \cdot \mathrm{m}^{2}$ (equivalent to $d=1 \mathrm{~mm}, \lambda=0.5 \mathrm{~W} / \mathrm{m} \cdot \mathrm{K}$ ), while the aft surface temperature is $1256 \mathrm{~K}$.

\section{Conclusions}

An innovative deployable heat shield design concept is proposed, which utilises centrifugal force generated by autorotation to deploy the structure under aerodynamic load. As demonstrated by simulations, the autorotation and deployment angle regulates each other and achieves a passive control that lets the heat shield reach an equilibrium deployment state.

Furthermore, a baseline vehicle is designed. This vehicle has a conventional deployable first stage that is used to induce initial deployment and improve the performance of the second stage. The flexible second stage is centrifugally deployed and has a shape based on an origami pattern to initiate autorotation while achieving compact packaging and shape morphing. 
Analytical equations are derived to characterise the structural dynamic behaviours of the second stage, which have satisfactory agreement with numerical $\mathrm{FE}$ and trajectory simulations. It is shown that at higher altitudes $(>30 \mathrm{~km})$, the heat shield always reaches near-fully deployed conditions, allowing it to effectively generate aerodynamic drag and survive the aerothermodynamic heating. Meanwhile, oscillations associated with deploying-folding motion exists throughout the descent. However, the oscillation amplitude is generally insignificant $\left(< \pm 10^{\circ}\right)$, and the frequency $(0.1 \mathrm{~Hz}$ - $10 \mathrm{~Hz}$ ) can be designed to be away from spin rate or aerodynamic pitching frequency to prevent coupling.

In addition, the heat shield can be lightweight and compact as no thermal insulation is needed on the flexible components. This also means the flexible heat shield's temperature can be reduced as a result of aft surface radiative cooling. For instance, on the baseline vehicle a reduction of 100 $\mathrm{K}$ is feasible under the heat flux of $27 \mathrm{~W} / \mathrm{cm}^{2}$.

The presented research is a preliminary study where simplified models are used to to reveal the basic performance of the heat shield, especially the baseline behaviour associated with deployment. The limitations of the models include: the structural model assumes axisymmetrical deployment and no structural damping; the three degree-of-freedom trajectory simulation assumes zero angle of attack and ignores gyroscopic effect.

\section{References}

[1] J. Cruz, J. Lingard, Aerodynamic decelerators for planetary exploration: past, present, and future, in: AIAA Guidance, Navigation, and Control Conference and Exhibit, AIAA paper 2006-6792, 2006.

[2] B. P. Smith, C. L. Tanner, M. Mahzari, I. G. Clark, R. D. Braun, F. M. Cheatwood, A historical review of inflatable aerodynamic decelerator technology development, in: Aerospace Conference, 2010 IEEE, IEEE, 2010, pp. 1-18.

[3] R. D. Braun, R. M. Manning, Mars exploration entry, descent, and landing challenges, Journal of Spacecraft and Rockets 44 (2) (2007) 310-323.

[4] B. Steinfeldt, J. Theisinger, A. Korzun, I. Clark, M. Grant, R. Braun, High mass mars entry, descent, and landing architecture assessment, in: AIAA SPACE 2009 Conference \& Exposition, AIAA paper 2009-6684, 2009.

[5] B. G. Drake, S. J. Hoffman, D. W. Beaty, Human exploration of mars, design reference architecture 5.0, in: Aerospace Conference, 2010 IEEE, IEEE, 2010, pp. 1-24.

[6] V. Carandente, R. Savino, New concepts of deployable de-orbit and re-entry systems for cubesat miniaturized satellites, Recent Patents on Engineering 8 (1) (2014) 2-12.

[7] B. Chan, N. Bauer, J. R. Juneau, S. Stout, K. Masuyama, D. Spencer, Recovery of in-space cubesat experiments (RICE) project, in: Seventh International Planetary Probe Workshop, Barcelona, Spain, 2010.

[8] G. Zuppardi, R. Savino, G. Mongelluzzo, Aero-thermo-dynamic analysis of a low ballistic coefficient deployable capsule in earth re-entry, Acta Astronautica 127 (2016) 593-602. 
[9] I. G. Clark, A. L. Hutchings, C. L. Tanner, R. D. Braun, Supersonic inflatable aerodynamic decelerators for use on future robotic missions to mars, in: Aerospace Conference, 2008 IEEE, IEEE, 2008, pp. 1-17.

[10] H. Bohon, M. Mikulas, M, Development status of attached inflatable decelerators., Journal of Spacecraft and Rockets 6 (6) (1969) 654-660.

[11] C. Tanner, J. Cruz, R. Braun, Structural verification and modeling of a tension cone inflatable aerodynamic decelerator, in: 51st AIAA/ASME/ASCE/AHS/ASC Structures, Structural Dynamics, and Materials Conference 18th AIAA/ASME/AHS Adaptive Structures Conference 12th, AIAA paper 2010-2830, 2010.

[12] A. Mastropietro, J. Kempenaar, M. Redmond, M. Pauken, W. Ancarrow, First test flight thermal performance of the low density supersonic decelerator (LDSD) supersonic flight dynamics test (SFDT) vehicle, in: 45th International Conference on Environmental Systems, 2015.

[13] D. Litton, D. Bose, F. Cheatwood, S. Hughes, H. Wright, M. Lindell, S. Derry, A. Olds, Inflatable re-entry vehicle experiment IRVE-4 overview, in: 21st AIAA Aerodynamic Decelerator Systems Technology Conference and Seminar, AIAA paper 2011-2580, 2011.

[14] D. Wilde, S. Walther, K. Pitchadze, S. Alexsaschkin, D. Vennemann, L. Marraffa, Flight test and ISS application of the inflatable reentry and descent technology (IRDT), Acta Astronautica $51(1-9)(2002) 83-88$.

[15] M. Wiegand, H. Konigsmann, A small re-entry capsule-BREM-SAT 2, in: 10th AIAA/USU Small Satellite Conference, Logan., Volume 1, 1996.

[16] D. L. Akin, The parashield entry vehicle concept-basic theory and flight test development, in: 4th AIAA/USU Small Satellite Conference, Logan, UT, Aug. 27-30, 1990, Proceedings. (A91-27376 10-18). Logan, Vol. 1, 1990.

[17] E. Stern, M. Barnhardt, E. Venkatapathy, G. Candler, D. Prabhu, Investigation of transonic wake dynamics for mechanically deployable entry systems, in: Aerospace Conference, 2012 IEEE, IEEE, 2012, pp. 1-10.

[18] E. Venkatapathy, K. Hamm, I. Fernandez, J. Arnold, D. Kinney, B. Laub, A. Makino, M. McGuire, K. Peterson, D. Prabhu, et al., Adaptive deployable entry and placement technology (ADEPT): a feasibility study for human missions to mars, in: 21st AIAA Aerodynamic Decelerator Systems Technology Conference and Seminar, AIAA paper 2011-2608, 2011.

[19] R. Detra, A. Kantrowitz, F. Riddell, P. Rose, The drag brake manned satellite system, in: Xth International Astronautical Congress London 1959/X. Internationaler Astronautischer Kongress/Xe Congrès International dAstronautique, Springer, 1960, pp. 728-747.

[20] M. Berzeri, A. A. Shabana, Study of the centrifugal stiffening effect using the finite element absolute nodal coordinate formulation, Multibody System Dynamics 7 (4) (2002) 357-387.

[21] V. Koshelev, V. Melnikov, Large space structures formed by centrifugal forces, CRC Press, 1998. 
[22] J. M. Hedgepeth, Dynamics of a large spin-stiffened deployable paraboloidal antenna, Journal of Spacecraft and Rockets 7 (9) (1970) 1043-1048.

[23] S. Nakasuka, T. Funane, Y. Nakamura, Y. Nojiri, H. Sahara, F. Sasaki, N. Kaya, Sounding rocket flight experiment for demonstrating furoshiki satellite for large phased array antenna, Acta Astronautica 59 (1) (2006) 200-205.

[24] M. Gärdsback, G. Tibert, Deployment control of spinning space webs, Journal of guidance, control, and dynamics 32 (1) (2009) 40-50.

[25] G. Kiper, E. Soylemez, Deployable space structures, in: Recent Advances in Space Technologies, 2009. RAST'09. 4th International Conference on, IEEE, 2009, pp. 131-138.

[26] C. H. Wolowicz, J. Brown Jr, W. P. Gilbert, Similitude requirements and scaling relationships as applied to model testing, Technical Report TP-1435, NASA.

[27] J. M. Gere, Mechanics of materials, sixth ed., Thomson Learning, Belmont, 2004.

[28] W. Wade, A. Sinha, R. Singh, Study of wrap-rib antenna design, Technical Report CR-162929, NASA.

[29] S. Hughes, J. Ware, J. Del Corso, R. Lugo, Deployable aeroshell flexible thermal protection system testing, in: 20th AIAA Aerodynamic Decelerator Systems Technology Conference and Seminar, AIAA paper 2009-2926, 2009.

[30] D. Coatta, D. Jurewicz, B. Tutt, T. Rivellini, I. Clark, Development and testing of an 8 meter isotensoid supersonic inflatable aerodynamic decelerator, in: 22nd AIAA Aerodynamic Decelerator Systems Conference, AIAA paper 2013-1328, 2013.

[31] P. Wercinski, Adaptable deployable entry and placement technology (ADEPT), Oral/Visual Presentation ARC-E-DAA-TN31961, NASA.

[32] P. Gallais, Atmospheric re-entry vehicle mechanics, Springer Science \& Business Media, 2007.

[33] A. Viviani, G. Pezzella, Aerodynamic and aerothermodynamic analysis of space mission vehicles, Springer, 2015.

[34] E. H. Hirschel, et al., Basics of aerothermodynamics, Springer, 2005.

[35] H. Wright, A. Cutright, J. Corliss, W. Bruce, D. Trombetta, A. Mazaheri, M. Coleman, A. Olds, S. Hancock, Heart flight test overview, in: 9th International Planetary Probe Workshop, 2012, pp. 16-22.

[36] D. Jurewicz, L. Lichodziejewski, B. Tutt, B. Gilles, G. Brown, Application of inflatable aeroshell structures for entry descent and landing, in: Aerospace Conference, 2013 IEEE, IEEE, 2013, pp. 1-10. 